\title{
Gravitational waves from deflagration bubbles in first-order phase transitions
}

\author{
Ariel Mégevand* \\ Departamento de Física, Facultad de Ciencias Exactas y Naturales, \\ Universidad Nacional de Mar del Plata, \\ Deán Funes 3350, (7600) Mar del Plata, Argentina
}

\begin{abstract}
The walls of bubbles in a first-order phase transition can propagate either as detonations, with a velocity larger than the speed of sound, or deflagrations, which are subsonic. We calculate the gravitational radiation that is produced by turbulence during a phase transition which develops via deflagration bubbles. We take into account the fact that a deflagration wall is preceded by a shock front which distributes the latent heat throughout space and influences other bubbles. We show that turbulence can induce peak values of $\Omega_{G W}$ as high as $\sim 10^{-9}$. We discuss the possibility of detecting at LISA gravitational waves produced in the electroweak phase transition with wall velocities $v_{w} \lesssim 10^{-1}$, which favor electroweak baryogenesis.
\end{abstract}

\section{INTRODUCTION}

One of the possible outcomes of a cosmological phase transition is gravitational radiation. Although it is difficult to detect, gravitational radiation provides a direct probe of the phase transition dynamics, since it propagates freely until the present epoch. Studies of the gravitational waves due to phase transitions at the electroweak scale and beyond [1] show that the signal may be within the sensitivity range of LISA and the second generation of space-based interferometers.

In a first-order phase transition, bubbles of the stable phase nucleate and expand, converting the high-temperature phase into the low-temperature one. Gravitational waves (GWs) are generated either by the collisions of bubbles [2, 3, 4, 5] or by the turbulence that is produced in the plasma due to the motion of bubble walls [3, 4, [5, 6, 7, 8, ,9, 10]. As bubbles expand, latent heat is released at the phase boundary. Part of this energy raises the temperature of the plasma, and another part is converted to bulk motions of the fluid. For phase transitions in the early Universe, the Reynolds number is large enough for the wall motion to produce turbulence. In general, the amplitude of GWs coming from turbulence dominates over bubble collisions.

The bubble wall can propagate via two modes, namely, detonation and deflagration. For a detonation, the phase transition front moves faster than the speed of sound $c_{s}$, whereas a deflagration front is subsonic. GWs produced by detonations have been extensively investigated [1, 2, 3, 4, 5, 6, 7, 8]. In this case, the bubble wall velocity $v_{w}$ depends only on the ratio of the latent heat to the total energy density, $\alpha[11]$. Since $v_{w}>c_{s}$, no signal precedes the detonation front. Hence, the dynamics of a wall is not influenced by other bubbles walls (except in the collision regions). Furthermore, the kinetic energy injected into the

*Member of CONICET, Argentina; megevand@mdp.edu.ar 
fluid is concentrated in a thin region behind the wall. This simplifies the integration of the kinetic-energy density profile. Besides, the temperature in the supercooled phase outside the bubbles decreases due to the expansion of the Universe. As a consequence, the nucleation rate $\Gamma$ increases exponentially. This justifies modeling it by $\Gamma=\Gamma_{0} e^{\beta t}$, which corresponds to linearizing the time dependence of the exponent. Hence, $\beta^{-1}$ is the only time scale in the problem and determines the duration of the phase transition, $\Delta t \sim \beta^{-1}$, and the mean bubble separation, $d \sim v_{w} \beta^{-1}$. Thus, the final result depends only on the parameters $\alpha$ and $\beta$. These features simplify the calculation of GWs.

The case of deflagrations is more difficult. In contrast to detonations, the deflagration front velocity depends on the viscosity of the plasma and on the pressure difference between phases, which in turn depends on the amount of supercooling. The wall propagates at a subsonic velocity and is preceded by a supersonic shock front which affects other bubbles. The shock wave distributes the latent heat injected by the wall throughout space, causing a reheating of the Universe and bulk motions of the fluid far away from the wall. For these reasons, the case of deflagrations has not received as much attention as the detonation case. In Ref. [4] an analytic expression is derived for the signal from bubble collisions. However, in that work the fluid velocity is left as a free parameter. Deflagrations have also been considered numerically in Ref. [5].

The spectrum of GWs for the deflagration case thus depends on several parameters, which are difficult to estimate in a specific model. As a consequence, the results for detonations have often been used to investigate the GW production in different phase transitions (see e.g. [1]), disregarding the fact that the phase transition may actually proceed via deflagration bubbles. Notice, indeed, that this is likely the general case. For instance, for the electroweak phase transition, estimations of the wall velocity give in general subsonic values $v_{w} \sim 10^{-2}-$ $10^{-1}[12]$, which are favorable for electroweak baryogenesis [13].

The aim of this work is to address the case of deflagration bubbles with a realistic approach. Thus, we take into account the fact that the shock waves coming from a bubble wall influence other bubbles. In section \we describe the dynamics of deflagration bubbles. One important feature is that, as a consequence of reheating, the nucleation rate turns-off and the bubble wall decreases during bubble expansion. Therefore, the approximation $\Gamma \propto e^{\beta t}$ cannot be used in this case. Furthermore, the smaller velocities at the collision time make bubble collisions to be suppressed relative to turbulence as a source of GWs. In section III] we consider the velocity spectrum of the turbulent fluid that arises from the motion of deflagration walls, and in section IV we follow the approach of Ref. [8] to derive the gravitational radiation produced by turbulence in the deflagration case. In section $\nabla$ we estimate the dependence of the amplitude and peak frequency on the latent heat, the bubble size, and the wall velocity. We show that for a strong enough electroweak phase transition, GWs from deflagration bubbles may be detected at LISA. Our conclusions are summarized in section VI.

\section{PHASE TRANSITION DYNAMICS}

A phase transition of the Universe occurs when the free energy of a system depends on an order parameter $\phi$ (in general a Higgs field), which develops a non-zero value. For a first-order phase transition, there is a temperature range in which the free energy $\mathcal{F}(\phi, T)$ has two minima separated by a barrier. Generally, the high-temperature minimum is $\phi=$ 0 , corresponding to the symmetric phase, and the low-temperature one is $\phi_{m}(T) \neq 0$, 
corresponding to the broken symmetry phase. At the critical temperature $T_{c}$, the two minima $\phi=0$ and $\phi=\phi_{m}$ have the same free energy. Below this temperature, $\phi_{m}(T)$ becomes the global minimum. At a certain temperature $T=T_{0}$, the barrier disappears and $\phi=0$ becomes a maximum of the free energy.

The energy density $\rho$ and entropy density $s$ are related to the free energy density by $\rho=T s+\mathcal{F}$ and $s=-d \mathcal{F} / d T$. We assume that the phase with $\phi=0$ is composed of radiation and false vacuum, i.e., $\mathcal{F}(0, T) \equiv \mathcal{F}_{+}(T)=\rho_{\Lambda}-\rho_{R} / 3$, where $\rho_{\Lambda}$ is the zerotemperature energy density of the false vacuum, $\rho_{\Lambda}=\mathcal{F}(\phi=0, T=0)=V(\phi=0)$, with $V(\phi)$ the zero-temperature effective potential, and $\rho_{R}$ is the energy density of radiation, $\rho_{R}=g_{*} \pi^{2} T^{4} / 30$, where $g_{*}$ is the number of relativistic degrees of freedom. Thus, for the high-temperature phase we have $\rho_{+}=\rho_{\Lambda}+\rho_{R}$, and for the low-temperature phase we have $\rho_{-}=-T \mathcal{F}_{-}^{\prime}+\mathcal{F}_{-}$. The pressure is given by $p=-\mathcal{F}$. Hence, at $T=T_{c}$ both phases have the same pressure. The latent heat $l$ is defined as the energy density discontinuity $l=\Delta \rho=\rho_{+}\left(T_{c}\right)-\rho_{-}\left(T_{c}\right)$. Thus, $l=T_{c} \Delta s=-T_{c} \Delta \mathcal{F}^{\prime}$. We note that in previous works on GW generation the latent heat is sometimes confused with the false vacuum energy density (or even with the free energy density). In fact, notice that in general $l$ can be anywhere in the range $0<l<\rho_{\Lambda}+\rho_{R}\left(T_{c}\right)$. On the other hand, it can be shown that $\rho_{\Lambda}$ is bounded by $0<\rho_{\Lambda}<\rho_{R}\left(T_{c}\right) / 3$ (assuming that the vacuum energy vanishes at $T=0$ ) [14].

The nucleation and growth of bubbles has been extensively investigated (see e.g. [11, 12, 13, 14, 15, 16, 17, 18, 19, 20, 21]). According to the standard picture, bubbles of the stable phase nucleate with a rate [15]

$$
\Gamma \approx T^{4} e^{-S_{3} / T}
$$

where $S_{3}(T)$ is the three-dimensional instanton action, which coincides with the free energy of a critical bubble. The nucleation rate is extremely sensitive to the temperature in the range $T_{0}<T<T_{c}$. At the critical temperature the radius $R_{c}$ of the critical bubble becomes infinite, so $S_{3}=\infty$ and $\Gamma=0$. In contrast, at $T=T_{0}$ the radius vanishes, so $S_{3}=0$ and $\Gamma \sim T_{c}^{4}$, which is an extremely large rate in comparison to $H^{4} \sim\left(T_{c}^{2} / M_{P}\right)^{4}$. Thus, bubbles begin to nucleate at an intermediate temperature $T_{\Gamma}$ for which $\Gamma \approx H^{4}$. To get an idea of the dependence of $\Gamma$ on $T$, consider the thin-wall approximation, which is valid near the critical temperature. In this case we can write $S_{3}=-\Delta \mathcal{F} 4 \pi R_{c}^{3} / 3+\sigma 4 \pi R_{c}^{2}$, where $\sigma$ is the surface tension of the bubble wall. Then we obtain the critical radius $R_{c}=2 \sigma / \Delta \mathcal{F}$ and the action $S_{3}=16 \pi \sigma^{3} / 3(\Delta \mathcal{F})^{2}$. For $T \approx T_{c}$ we can also approximate the free energy difference by $\Delta \mathcal{F} \approx l\left(T_{c}-T\right) / T_{c}$. Thus, we obtain

$$
\frac{S_{3}(T)}{T} \approx \frac{16 \pi \sigma^{3} T_{c}}{3 l^{2}\left(T_{c}-T\right)^{2}}
$$

Once a bubble is nucleated, it begins to grow. The bubble radius rapidly becomes much larger than $R_{c}$. Due to the viscosity of the plasma, the bubble walls immediately reach a terminal velocity $v_{w}$ which is determined by the pressure difference $\Delta p=-\Delta \mathcal{F}$ and the friction with the surrounding particles. It can be approximated by $v_{w}(T)=\Delta \mathcal{F}(T) / \eta$, where $\eta$ is a friction coefficient. Using again the linear approximation for $\Delta \mathcal{F}$ we obtain

$$
v_{w} \approx l\left(T_{c}-T\right) / \eta T_{c}
$$

For strong phase transitions, the latent heat $l$ and the amount of supercooling $\left(T_{c}-T_{\Gamma}\right) / T_{c}$ will be considerable. Notice, however, that the velocity depends also on the viscosity and can be subsonic even in this case. 
We shall assume that the wall propagates as a deflagration front. Therefore, the wall velocity is lower than the speed of sound in the relativistic plasma, $c_{s}=\sqrt{1 / 3}$. In this case, a shock front precedes the wall with a velocity $v_{s h} \gtrsim c_{s}$. Consequently, the shock front of a bubble soon influences other bubbles. For $v_{w} \ll c_{s}$, the latent heat is transmitted away from the wall and is quickly distributed throughout space. This effect can be taken into account by considering a homogeneous reheating of the plasma as latent heat is being injected [18]. Even with this simple approximation, the evolution of the phase transition must be computed numerically. Therefore, it is not straightforward to find relations between thermodynamic parameters such as $l$ or $\sigma$ and the quantities that characterize the dynamics, such as the duration of the phase transition or the bubble number density.

Nevertheless, as we shall see, the general features of the dynamics of slow bubble walls provide a good deal of information for the computation of gravitational waves. As a consequence of reheating, the free energy difference $\Delta \mathcal{F}(T)$ decreases and, according to Eq. (3)), the bubble expansion slows down. This effect will be important if the latent heat is comparable to the difference $\delta \rho=\rho_{R}\left(T_{c}\right)-\rho_{R}\left(T_{\Gamma}\right)$. Thus, if $l \gg \delta \rho$, the temperature will get very close to the critical one ${ }^{1}$. Then, the velocity will decrease significantly and a long phase coexistence stage will take place before the transition completes [14, 19, 20]. In any case, due to the exponential dependence of the nucleation rate on temperature, bubble nucleation turns off as soon as temperature begins to raise [20, 21]. Indeed, notice that the temperature $T(t)$ has a minimum, which separates the supercooling and reheating stages. According to Eqs. (1) and (2), the nucleation rate has a maximum at that time, and due to the extreme dependence of $\Gamma$ on $T_{c}-T$, the maximum must be a sharp peak. Hence, the minimum temperature gives the nucleation temperature $T_{\Gamma}$. Most bubbles are created in a very short time interval $\delta t_{\Gamma}$ around the time $t_{\Gamma}$ corresponding to this temperature.

For $t>t_{\Gamma}$, the number density of bubbles $n_{b}$ remains constant. The final size of bubbles is given by $d \sim n_{b}^{-1 / 3}$. Most bubbles begin to expand at $t \approx t_{\Gamma}$ with velocity $v_{w} \equiv v_{i}$. During reheating the wall velocity decreases, and by the time bubbles percolate $v_{w}$ will be in general much smaller than the initial velocity $v_{i}$. As a consequence, the GW signal from bubble collisions will be too low. Therefore, we will consider only GWs from turbulence. The main turbulence will be generated during the reheating stage, when $v_{w}$ is still close to $v_{i}$. The frequency and amplitude of the GWs will depend on the dynamics of the phase transition, which is involved. However, as we shall see, the result will be essentially determined by a few parameters.

\section{TURBULENCE FROM DEFLAGRATION BUBBLES}

Turbulence from stirring in cosmological phase transitions has been extensively studied [5, 6, 7, 8, 9, 10]. When turbulence is fully developed, a cascade of energy is established from larger to smaller length scales, as eddies of each size break into smaller ones. We define the energy dissipation rate per unit enthalpy for a given momentum scale $k=2 \pi / L$,

$$
\left.\varepsilon_{k} \equiv \frac{1}{w} \frac{d \rho_{\text {turb }}}{d t}\right|_{\text {in }},
$$

\footnotetext{
1 This is quite generally the case. For strong phase transitions, we have $l \sim \rho \gg \delta \rho$. For weaker phase transitions, the latent heat is smaller, but so is $\delta \rho$.
} 
where $w=\rho+p$ is the enthalpy density, $\rho$ is the total energy density of the fluid, $p$ is the pressure, and $\rho_{\text {turb }}=w\left\langle v^{2}\right\rangle / 2$ is the kinetic energy density of turbulence. Since the phase transition occurs in the radiation dominated epoch, we will make for simplicity the usual $\operatorname{assumption}^{2} \rho \approx \rho_{R}$. Hence, $p \approx p_{R}=\rho_{R} / 3$ and $w \approx w_{R}=4 / 3 \rho_{R}$. If the process is stationary, $\varepsilon_{k}$ gives the rate at which turbulent energy is received at the scale $k$ from higher length scales (and transferred to smaller length scales). If the external source stirs the fluid at a single scale $k_{S}=2 \pi / L_{S}$, we have a constant rate $\varepsilon_{k} \equiv \varepsilon$ for scales $L<L_{S}$ [7]. In this case the turbulent energy in the cascade is characterized by the Kolmogoroff spectrum

$$
E(k) \equiv \frac{1}{w} \frac{d \rho_{\mathrm{turb}}}{d k} \approx \varepsilon^{2 / 3} k^{-5 / 3}
$$

The cascade stops at the damping scale $k_{D}=2 \pi / L_{D}$, with $L_{D} \ll L_{S}$, at which the fluid viscosity dissipates the injected energy into heat.

As usual, we assume that the fluid is incompressible and statistically isotropic and homogeneous. Then, the Fourier transform of the velocity has the two point correlation function

$$
\left\langle v_{i}(\mathbf{k}) v_{j}^{*}(\mathbf{q})\right\rangle=(2 \pi)^{3} \delta^{3}(\mathbf{k}-\mathbf{q})\left(\delta_{i j}-\hat{\mathbf{k}}_{i} \hat{\mathbf{k}}_{j}\right) P(k),
$$

where the angular brackets mean a statistical average. Notice that $\left\langle\mathbf{v}^{2}(\mathbf{x})\right\rangle$ gives the kinetic energy density per unit enthalpy density of the fluid. Therefore, the velocity spectrum $P(k)$ can be related to the energy spectrum $E(k)$. The relation is $E(k)=k^{2} P(k) / 2 \pi^{2}$ [22]. From Eq. (5), we have

$$
P(k) \approx \pi^{2} \varepsilon^{2 / 3} k^{-11 / 3}
$$

in the inertial range $k_{S} \ll k \ll k_{D}$. The power spectrum $P(k)$ beyond this range was obtained in Ref. [8] from an ansatz for the real-space correlation function. We have

$$
P(k) \approx 2 \pi\left\langle v^{2}\right\rangle \begin{cases}\frac{2}{765} L_{S}^{5} k^{2} & \text { for } k \ll k_{S} \\ \frac{55}{81} \sqrt{3} \Gamma\left(\frac{2}{3}\right) L_{S}^{-2 / 3} k^{-11 / 3} & \text { for } k_{S} \ll k \ll k_{D} \\ 0 & \text { otherwise, }\end{cases}
$$

and the normalization of the spectrum is given by

$$
\left\langle v^{2}\right\rangle \approx\left(\varepsilon L_{S}\right)^{2 / 3}
$$

which corresponds to the fluid velocity on the largest scale $L_{S}$ (see below).

For stationary turbulence, the energy dissipation rate in Eq. (4) must equal the power that is injected by the source. Thus, for the case of expanding bubbles we have

$$
\varepsilon=\frac{1}{w} \kappa l \frac{d f_{b}}{d t}
$$

where $f_{b}$ is the fraction of volume occupied by bubbles and $\kappa$ is an efficiency factor which quantifies the fraction of latent heat that goes into kinetic energy of the fluid (a fraction $1-\kappa$

\footnotetext{
${ }^{2}$ In fact, the energy density at the beginning of the phase transition is $\rho_{+}=\rho_{R}+\rho_{\Lambda}$. For a thermal phase transition the false-vacuum energy is bounded by $\rho_{\Lambda}<\rho_{R} / 3$ and is in general $\rho_{\Lambda} \ll \rho_{R}$ [14]. Part of $\rho_{+}$ is liberated as latent heat, so that at the end of the transition we have $\rho_{-}=\rho_{+}-l$.
} 
goes into thermal energy and causes reheating of the plasma). The shock wave in front of the wall sets the fluid moving outward with a velocity $v_{f}$. Hence, the injected kinetic-energy density is

$$
\rho_{\mathrm{kin}}=\frac{1}{2} w v_{f}^{2} .
$$

As the phase transition front moves a distance $v_{w} \delta t$, the energy released is proportional to $l v_{w} \delta t$. Assuming for simplicity a constant fluid velocity up to a distance $\sim c_{s} t$, the kinetic energy injected in the time $\delta t$ will be proportional to $\rho_{\text {kin }} c_{s} \delta t$. Thus, we have

$$
\kappa=\frac{\rho_{\mathrm{kin}} c_{s}}{l v_{w}} .
$$

We still need to determine the fluid velocity $v_{f}$ appearing in Eq. (11). The conservation of energy and momentum $\partial_{\mu} T^{\mu \nu}=0$ can be applied to the wall discontinuity to obtain relations for the quantities on both sides of the wall. Assuming a stationary solution and non-relativistic velocities, one obtains the equation (see e.g. [17, 22])

$$
w_{+} v_{+}=w_{-} v_{-},
$$

where $w$ is the enthalpy, $v$ is the velocity of the fluid in the rest frame of the phase transition front, and the plus and minus signs stand for the high- and low-temperature phase regions, respectively. In the rest frame of the center of the bubble, the fluid inside the bubble is at rest, the wall velocity is $v_{w}=-v_{-}$, and the fluid velocity in front of the wall is $v_{f}=v_{+}-v_{-}$. Using Eq. (13) we obtain

$$
v_{f}=\frac{w_{+}-w_{-}}{w_{+}} v_{w} \approx \frac{\Delta \rho+\Delta p}{w_{R}} v_{w},
$$

where $\Delta \rho$ and $\Delta p$ are the energy-density and pressure differences across the wall. Notice that, even if the temperature is homogeneous, $\rho(T)$ and $p(T)$ are different in each phase. $\Delta \rho(T)$ and $\Delta p(T)$ depend on the amount of supercooling. For simplicity, we will assume that the temperature remains close to the critical one, so that $\Delta p \approx 0$ and the energy density discontinuity is given by the latent heat, $\Delta \rho\left(T_{c}\right) \equiv l$. Therefore, we have

$$
v_{f} \approx\left(l / w_{R}\right) v_{w} .
$$

From Eqs. (11), (12), and (15) we obtain

$$
\kappa=\frac{1}{2}\left(l / w_{R}\right) v_{w} c_{s}
$$

Thus, Eq. (10) gives

$$
\varepsilon=(\kappa l / w) \dot{f}_{b}=(1 / 2) v_{w} c_{s} \alpha^{2} \dot{f}_{b},
$$

where we have defined the ratio of the latent heat to the enthalpy density, $\alpha \equiv l / w_{R}$.

For the validity of the Kolmogoroff spectrum, it is important that the energy is injected at a single scale $L_{S}$. For the kind of phase transitions we are interested in, all the bubbles are formed in a short interval $\delta t_{\Gamma}$ around the time $t_{\Gamma}$. Hence, the number density of bubbles $n_{b}$ is set at $t \approx t_{\Gamma}$. For $t>t_{\Gamma}$, the number of bubbles remains constant, and the wall velocity $v_{w}(T(t))$ is the same for all bubbles. The bubble radius is $R(t)=\int_{t_{\Gamma}}^{t} v_{w}\left(t^{\prime}\right) d t^{\prime}$, and the 
distribution of sizes has a small width $\delta R \approx v_{w}\left(t_{\Gamma}\right) \delta t_{\Gamma} \ll R$. At the moment of collision the bubble size is given by the distance between bubble centers, $d \sim n_{b}^{-1 / 3}$.

In fact, turbulence begins as soon as the shock fronts collide. The first shocks are emitted at $t \approx t_{\Gamma}$ and collide when they reach the size $d$, after a time $\sim d / c_{s}$. This time is much smaller than the total duration $\Delta t$ of the phase transition. Indeed, for deflagrations the wall velocity is $v_{w}<c_{s}$. In addition, as explained in section II, $v_{w}$ decreases significantly from its initial value $v_{i}$. Therefore, we have $\Delta t \gg d / v_{i}>d / c_{s}$. Moreover, as mentioned in section I, in the general case the initial velocity may be already $v_{i} \ll c_{s}$, as suggested by the electroweak case. When shock fronts collide, they lose their spherical symmetry. Bubbles continue expanding, and their walls continue injecting energy into the fluid until all space is filled up. Thus, turbulence begins very soon, at $t \approx t_{\Gamma}$, and remains until the phase transition is complete. As bubbles expand, the latent heat is taken away at the speed of sound, always stirring the fluid at the same scale $L_{S} \sim d$. Hence, it is reasonable to assume a Kolmogoroff spectrum with $k_{S} \sim 2 \pi / d$. Notice that in the detonation case, in contrast, there will be much more small bubbles (with $L \ll d$ ) than large ones (with $L \sim d$ ), due to the exponentially increasing nucleation rate $\Gamma=\Gamma_{0} e^{\beta t}$. Thus, the small bubbles will in principle modify the Kolmogoroff spectrum in the detonation case.

It is important to estimate the turnover time scale of an eddy, $\tau_{L} \approx L / v_{L}$, where $v_{L}$ is the characteristic fluid velocity on a length scale $L$. The rate at which an eddy breaks into smaller ones is usually assumed to be roughly $\sim \tau_{L}^{-1}$. Thus, the rate at which energy is transferred in the cascade is $\varepsilon \sim v_{L}^{2} \tau_{L}^{-1}$. Therefore, we have $v_{L} \sim(\varepsilon L)^{1 / 3}$ and $\tau_{L} \sim \varepsilon^{-1 / 3} L^{2 / 3}$. The turnover frequency is given by $\omega_{k}=\omega_{S}\left(k / k_{S}\right)^{2 / 3}$, with $\omega_{S}=v_{L_{S}} / L_{S}$.

Both $v_{L}$ and $\tau_{L}$ increase with $L$. The maximum length scale is the characteristic scale of the source $L_{S} \sim d$. In general, $d$ is well inside the horizon [14, 18, 21]. Hence, the time scale for the establishment of a cascade, which is on the order of the maximum turnover time $\tau_{d}$, will be at most on the order of the Hubble time. This justifies neglecting the expansion of the Universe in the description of turbulence. On the other hand, it is important to compare the characteristic turnover time with the duration of the phase transition. For the case of detonations, it has been shown that $\tau_{d}$ is always larger than $\Delta t$ (see e.g. [6, 8]). This result is more general. Indeed, since $\tau_{d} \sim d / v_{d}, \Delta t \sim d / v_{w}$, and the fluid velocity is always smaller than the wall velocity, we have $\tau_{d}>\Delta t$. Since the stirring source lasts less than $\tau_{d}$, turbulence is not stationary. Nevertheless, the cascade of energy develops, and it was argued [6] that, for the generation of gravitational waves, we can assume stationary turbulence with a duration $\sim \tau_{L_{S}}=\tau_{d}$.

\section{RELIC GRAVITATIONAL WAVES}

We aim to calculate the spectrum we would observe today for GWs originated in a cosmological phase transition at time $t=t_{*}$ and temperature $T=T_{*}$. As explained in Sec. III, in the deflagration bubble scenario the wall velocity is in general considerably smaller at the time of percolation than at the beginning of bubble expansion. Therefore, we expect a weak signal from bubble collisions. In view of that, we shall only consider the source provided by turbulence. The calculation of the GW spectrum from primordial turbulence has been improved in the last years [8, 9]. Here we will use the results of Ref. [8] (see the discussion in Sec. VI). In this section we briefly review the derivation and write down the results in terms of variables that are suitable for the deflagration bubble scenario.

For a stochastic background of gravitational waves, the spectrum is characterized by the 
quantity [23]

$$
\Omega_{G W}(f)=\frac{1}{\rho_{c}} \frac{d \rho_{G W}}{d \log f},
$$

where $\rho_{G W}$ is the energy density of the GWs, $f$ is the frequency, and $\rho_{c}$ is the critical energy density today, $\rho_{c}=3 H_{0}^{2} / 8 \pi G$. Thus, the gravitational wave energy density per unit logarithmic frequency is defined by the relation

$$
\rho_{G W}=\int \frac{d f}{f} \frac{d \rho_{G W}}{d \log f} .
$$

For a stochastic background, $d \rho_{G W} / d \log f$ is given by the ensemble average of the Fourier amplitudes of the tensor metric perturbation $h_{i j}$. The energy density of gravitational waves is

$$
\rho_{G W}(\mathbf{x}, t)=\frac{\left\langle\partial_{t} h_{i j}(\mathbf{x}, t) \partial_{t} h_{i j}(\mathbf{x}, t)\right\rangle}{16 \pi G},
$$

where the brackets denote the ensemble average. The source of $h_{i j}(\mathbf{x}, t)$ is the transverse and traceless piece of the stress-energy tensor, which for a turbulent plasma is given by $T_{i j}(\mathbf{x}, t)=w v_{i}(\mathbf{x}, t) v_{j}(\mathbf{x}, t)$. Thus, the source for tensor perturbations on each mode $\mathbf{k}$ is the anisotropic stress $\Pi_{i j}(\mathbf{k})$, which involves a convolution $\int d^{3} q v_{i}(\mathbf{q}, t) v_{j}(\mathbf{k}-\mathbf{q}, t)$ (see [8] for details). Therefore, the energy density spectrum, which involves the average $\left\langle\Pi_{i j}(\mathbf{k}) \Pi_{i j}^{*}(\mathbf{q})\right\rangle$, can be related to the velocity spectrum $P(k)$ by means of Eq. (6) .

Notice that the velocity correlation function (6) does not oscillate in time. However, the gravitational radiation is produced by the turbulent eddies, which have a turnover frequency $\omega_{k}=v_{L} / L$, with $k=2 \pi / L$. The oscillatory behavior of the source, which is relevant for the generation of GWs, is lost in the statistical average. One can account for the turnover frequency by replacing $v_{i}(\mathbf{k}) \rightarrow v_{i}(\mathbf{k}) e^{i \omega_{k} t}$ for $k_{S}<k<k_{D}$. As a consequence, the source for $h_{i j}(\mathbf{k}, t)$ can be modeled as $e^{i 2 \bar{\omega} t} \Pi_{i j}(\mathbf{k}) \Theta\left(t-t_{\text {in }}\right) \Theta\left(t_{\text {fin }}-t\right)$, where $\bar{\omega}=\omega_{k}$ for $k>k_{S}, \bar{\omega}=\omega_{S}$ for $k<k_{S}$, and the Heaviside functions limit the source to the interval $t_{\text {in }}<t<t_{\text {fin }}$. We set $t_{\text {fin }}-t_{\text {in }}=\tau_{L_{S}}$ and $t_{\text {fin }}=t_{*}$. For $t>t_{*}$, the gravitational wave propagates freely, with the dispersion relation $\omega=k$. Hence, the frequency $f$ in Eq. (18) is given by the wave number $k$ of the source. The amplitude of the wave is proportional to $\Pi_{i j}(\mathbf{k})$ and depends on the frequency $\bar{\omega}$ of the source.

The spectrum of GWs is obtained from the quantity $\left\langle\dot{h}_{i j}(\mathbf{k}, t) \dot{h}_{i j}(\mathbf{q}, t)\right\rangle$. It is proportional to the dimensionless function

$$
A(\bar{\omega}, k)=\frac{\left|e^{i(2 \bar{\omega}-k) / \omega_{S}}-1\right|^{2}}{(k-2 \bar{\omega})^{2} L_{S}^{2}}+\frac{\left|e^{i(2 \bar{\omega}+k) / \omega_{S}}-1\right|^{2}}{(k+2 \bar{\omega})^{2} L_{S}^{2}}
$$

and to $\left\langle\Pi_{i j}(\mathbf{k}) \Pi_{i j}^{*}(\mathbf{q})\right\rangle$. The latter is a four-point spectral function of the velocity, and must be reduced in order to use the two-point function (6). This is usually done by using Wick's theorem, although the velocity field is not Gaussian. We have

$$
\left\langle\Pi_{i j}(\mathbf{k}) \Pi_{i j}^{*}(\mathbf{q})\right\rangle=w^{2} \delta(\mathbf{k}-\mathbf{q}) \int d^{3} p P(\mathbf{p}) P(|\mathbf{k}-\mathbf{p}|)\left(1+\gamma^{2}\right)\left(1+\beta^{2}\right),
$$

where $\gamma=\hat{\mathbf{k}} \cdot \hat{\mathbf{p}}, \beta=\hat{\mathbf{k}} \cdot \widehat{\mathbf{k}-\mathbf{p}}$. Analytical approximations exist for the integral in Eq. (22) for large and small scales [6, 24]. It can then be evaluated using Eqs. (8) and (91). 
Assuming radiation domination at $T=T_{*}$, the enthalpy $w$ in Eq. (22) can be approximated by $w_{R}=4 \rho_{R} / 3$, and the Hubble rate is given by $H_{*}^{2}=8 \pi G \rho_{R} / 3$. Finally, at $t=t_{*}$ we have ${ }^{3}$

$$
\rho_{G W}\left(k, t_{*}\right)=\frac{\rho_{R}\left(t_{*}\right)}{4 \pi}\left(H_{*} L_{S}\right)^{2}\left(\varepsilon L_{S}\right)^{4 / 3} \begin{cases}\frac{2}{13} A\left(\omega_{S}, k\right)\left(k L_{S}\right)^{3} & \text { for } k<k_{S} \\ A\left(\omega_{k}, k\right)\left(k L_{S}\right)^{-2 / 3} & \text { for } k_{S}<k<k_{D} \\ 0 & \text { otherwise. }\end{cases}
$$

The function $A(\bar{\omega}, k)$ gives a different spectral dependence whether the largest eddy velocity $v_{L_{S}}$ is below or above $1 / 2$, but the GW spectrum always peaks at $k=k_{S}$. For deflagrations the velocity of the fluid is smaller than the bubble wall velocity, which is smaller than that of sound $\left(c_{s} \approx 0.58\right)$. Thus, only in the limit in which both $v_{L_{S}} \approx v_{w}$ and $v_{w} \approx c_{s}$, we will have $v_{L_{S}}>1 / 2$. We shall deal with wall velocities $v_{w} \lesssim 0.1$. Therefore, we only consider the case $v_{L_{S}}<1 / 2$, for which the energy spectrum can be approximated by

$$
\rho_{G W}\left(k, t_{*}\right)=\frac{\rho_{R}\left(t_{*}\right)}{2 \pi}\left(H_{*} L_{S}\right)^{2}\left(\varepsilon L_{S}\right)^{4 / 3} \begin{cases}\left(k / k_{S}\right)^{3} / v_{L_{S}}^{2} & \text { for } k<2 v_{L_{S}} k_{S} \\ 4\left(k / k_{S}\right) & \text { for } 2 v_{L_{S}} k_{S}<k<k_{S} \\ 4\left(k / k_{S}\right)^{-8 / 3} & \text { for } k_{S}<k<k_{D} \\ 0 & \text { otherwise. }\end{cases}
$$

The GWs generated at time $t_{*}$ redshift due to the expansion of the Universe. The energy density scales like $a^{-4}$, and the frequency like $a^{-1}$. Therefore, the spectrum today is given by Eq. (24), with $\rho_{R}\left(t_{*}\right)$ replaced with $\rho_{R}\left(t_{0}\right)$ and the wave numbers replaced with the corresponding frequencies. Hence,

$$
\Omega_{G W}\left(f, t_{0}\right)=\frac{\Omega_{R}\left(t_{0}\right)}{2 \pi}\left(\frac{L_{S}}{H_{*}^{-1}}\right)^{10 / 3}\left(\frac{\varepsilon}{H_{*}}\right)^{4 / 3} \begin{cases}\left(f / f_{p}\right)^{3} / v_{L_{S}}^{2} & \text { for } f<2 f_{S} \\ 4\left(f / f_{p}\right) & \text { for } 2 f_{S}<f<f_{p} \\ 4\left(f / f_{p}\right)^{-8 / 3} & \text { for } f_{p}<f<f_{D} \\ 0 & \text { otherwise }\end{cases}
$$

where $\Omega_{R}\left(t_{0}\right)=\rho_{R} / \rho_{c} \approx 4.6 \times 10^{-5}$ [25], and $f_{S}, f_{p}, f_{D}$ are the redshifted frequencies corresponding respectively to the frequency of the largest eddies $f_{S *}=v_{L_{S}} L_{S}^{-1}$, the peak frequency $f_{p *}=L_{S}^{-1}$, and the dissipation frequency $f_{D *}=L_{D}^{-1}$. A frequency $f_{*}$ redshifted to today is given by $f_{0}=f_{*} a_{*} / a_{0}$. The ratio of the scale factor at $t_{*}$ to the scale factor today is

$$
\frac{a_{*}}{a_{0}} \approx 8 \times 10^{-16}\left(\frac{100}{g_{*}}\right)^{1 / 3} \frac{100 G e V}{T_{*}} .
$$

It is useful to express $f_{0}$ in terms of $f_{*} / H_{*}$. The Hubble rate $H_{*}=\sqrt{8 \pi G \rho_{R} / 3}$ can be written as

$$
H_{*} \approx 2 \times 10^{10} \mathrm{~Hz}\left(\frac{g_{*}}{100}\right)^{1 / 2}\left(\frac{T_{*}}{100 G e V}\right)^{2},
$$

where we have used the relations $G=M_{P l}^{-2}$, with $M_{P l}=1.22 \times 10^{19} \mathrm{GeV}$, and $1 \mathrm{GeV} \approx$ $1.5 \times 10^{24} \mathrm{~Hz}$. Therefore, the frequency today is given by

$$
f_{0}=1.6 \times 10^{-5} H z \frac{T_{*}}{100 G e V}\left(\frac{g_{*}}{100}\right)^{1 / 6} \frac{f_{*}}{H_{*}} .
$$

\footnotetext{
${ }^{3}$ In Ref. 8] the calculations were done using conformal time $\eta$ and comoving variables $\mathbf{k}, \mathbf{x}, L$, etc. Dimensionless combinations such as $H L$ are readily translated to physical variables, since the scale factor $a$ cancels out.
} 


\section{GRAVITATIONAL RADIATION FROM THE PHASE TRANSITION}

As we have seen, the scale $L_{S}$ is given by the bubble separation. Therefore, the peak frequency is

$$
f_{p}=1.6 \times 10^{-2} m H z\left(\frac{g_{*}}{100}\right)^{1 / 6} \frac{T_{*}}{100 G e V} \frac{H_{*}^{-1}}{d} .
$$

The distance $d$ depends on the dynamics of the phase transition. It can vary from values $d / H_{*}^{-1} \sim 10^{-5}$ for weakly first-order phase transitions, to values $d / H_{*}^{-1} \sim 10^{-1}$ for strongly first-order phase transitions (see e.g. [14, 18, 21] ). For $T_{*} \gtrsim 100 \mathrm{GeV}$, we see that millihertz frequencies (corresponding to the peak sensitivity of LISA) are obtained for relatively large values of the bubble size, $d / H_{*}^{-1} \gtrsim 10^{-2}$ (for $g_{*} \sim 100$ ).

Setting $L_{S}=d$ in Eq. (25) we obtain, for the maximum of the spectrum today,

$$
\left.\Omega_{G W}\right|_{\text {peak }}=3 \times 10^{-5}\left(\frac{d}{H_{*}^{-1}}\right)^{10 / 3}\left(\frac{\varepsilon}{H_{*}}\right)^{4 / 3}
$$

According to Eq. (17), the rate $\varepsilon$ at which energy is injected into the fluid is proportional to $v_{w} \dot{f}_{b}$. The speed of bubble expansion may vary considerably due to reheating during the phase transition. Nevertheless, we have seen that the turbulence which sources the gravitational radiation lasts longer than the phase transition. Hence, the generation of GWs is not affected by the details of the time dependence of $\varepsilon$, and we can use in Eq. (30) a mean value $\bar{\varepsilon}$ which involves a time average of $v_{w}(t) \dot{f}_{b}(t)$. A precise calculation of the parameters $d$ and $\bar{\varepsilon}$ requires a numerical computation of the phase transition. We will address such computation elsewhere [26]. Below, we find an approximation for $\dot{f}_{b}$ as a function of $v_{w}$ and $d$. The bubble separation $d$ also depends on the wall velocity. The larger the initial velocity $v_{i}$, the quicker the reheating and the sooner the turn-off of the nucleation rate. Roughly, we have $d \sim n_{b}^{-1 / 3} \propto v_{i}\left[20\right.$. Unfortunately, the number density of bubbles $n_{b}$ is extremely sensitive to the dynamics of the phase transition. This prevents any sensible analytical approximation for $d$ as a function of the parameters of the model.

The bubble expansion rate depends on the wall velocity and the bubble size. Roughly, $\dot{f}_{b} \sim F d^{2} \bar{v}_{w} / d^{3}=F \bar{v}_{w} / d$, where $F$ is a geometrical factor (for a spherical bubble, $F=4 \pi$ ), and $\bar{v}_{w}$ is the average wall velocity. In general, it will not be a good approximation to take a plain time average over the entire duration of the phase transition. The bubble expansion will slow down significantly due to reheating. Therefore, the turbulence that is generated while the wall velocity $v_{w}$ is close to its initial value $v_{i}$ will give the main contribution to $\Omega_{G W}$. Hence, we can use $v_{i}$ instead of $\bar{v}_{w}$ in the approximations above. Inserting Eq. (17) in Eq. (30) and using $v_{w} \sim v_{i}, \dot{f}_{b} \sim F v_{i} / d$, we have

$$
\left.\Omega_{G W}\right|_{\text {peak }} \sim 6 \times 10^{-6}\left(\alpha v_{i}\right)^{8 / 3} F^{4 / 3}\left(\frac{d}{H_{*}^{-1}}\right)^{2} .
$$

Again, we see that strong phase transitions favor detection at LISA, since the amplitude is maximized for large values of $d / H_{*}^{-1}$ (which also give millihertz frequencies). Weak phase transitions give smaller values of $d / H_{*}^{-1}$, and also smaller values of $v_{i}$ and $\alpha$. Therefore, we will have very low values of $\Omega_{G W}\left(f_{p}\right)$, together with high values of the frequency, $f_{p}>m H z$. In that case, the generated GWs will not be detected by LISA, but may be detected by space-based interferometers of second generation, such as BBO. 
Let us consider an electroweak phase transition at $T_{*} \approx 100 \mathrm{GeV}$, with $g_{*} \approx 100$, and set $F \sim 10$. The bubble separation $d$ depends on the extension of the Standard Model. For the frequency to be in the band that LISA is sensitive to, the phase transition should be strongly first-order, so that $d / H_{*}^{-1} \sim 10^{-2}$. Thus, for a wall velocity $v_{i} \sim 10^{-1} \mathrm{Eq}$. (31) gives $\Omega_{G W} \sim 10^{-11}$, provided that the latent heat is large enough (i.e., $\alpha \sim 1$ ). A large latent heat is consistent with a strongly first-order phase transition. This value of $\Omega_{G W}$ is just at the detection threshold of LISA. Notice, however, that this is an order-ofmagnitude estimate, and the result is sensitive to several parameters. For instance, the enthalpy difference $w_{+}-w_{-}$in Eq. (14) will give a larger efficiency factor if it is evaluated at the supercooling temperature $T_{\Gamma}<T_{c}$. For a phase transition at $T_{*}=1 T e V$, Eq. (29) gives the required frequency $f_{p} \sim m H z$ for a value $d / H_{*}^{-1} \sim 10^{-1}$. In this case, for $\alpha \sim 1$ and $v_{i} \sim 10^{-1}$ we have $\Omega_{G W} \sim 10^{-9}$. In Fig. 1 we plot the minimum value of $\alpha$ that is needed to achieve a peak value $\Omega_{G W}\left(f_{p}\right) \geq 10^{-11}$ with a peak frequency $f_{p}=1 \mathrm{mHz}$ for a phase ransition that takes place at $T=T_{*}$.

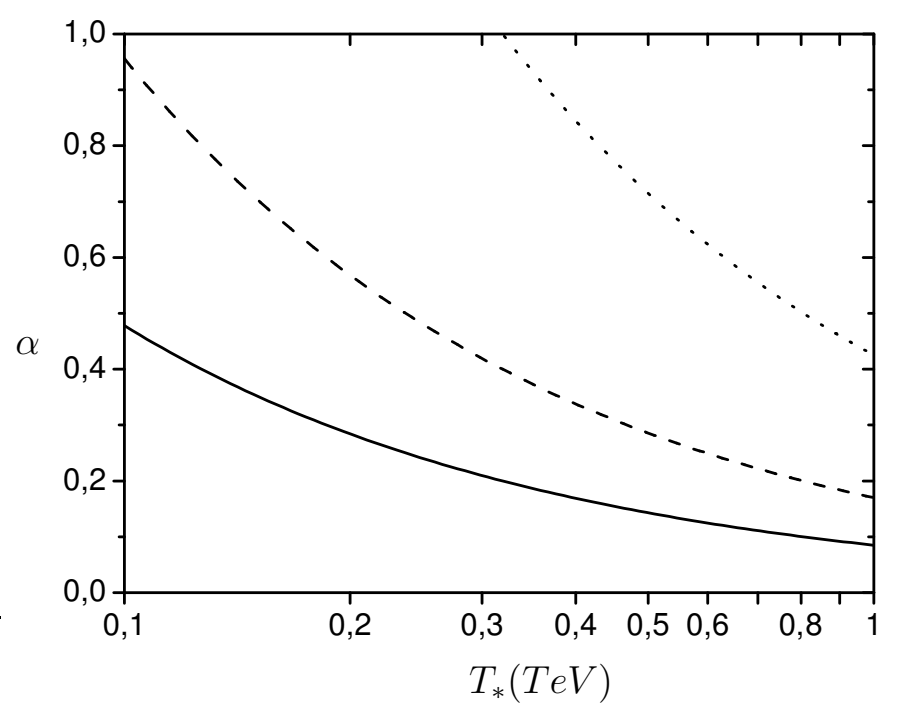

FIG. 1: The values of $\alpha$ and $T_{*}$ that give $f_{p}=1 \mathrm{mHz}$ and $\Omega_{G W}\left(f_{p}\right)=10^{-11}$, for $v_{i}=0.1$ (solid line), $v_{i}=0.05$ (dashed line) and $v_{i}=0.02$ (dotted line).

\section{CONCLUSIONS}

We have considered the production of gravitational waves in a first-order phase transition, due to the turbulence that arises from the motion of deflagration fronts. As we have seen, the dynamics of the phase transition is completely different from the case of detonations. The main difference is the fact that deflagration walls are preceded by shock fronts, which move much faster than them and influence other bubbles. This affects both the bubble expansion and the generation of GWs. Firstly, as soon as bubbles begin to nucleate and expand, the shock fronts collide and the spherical symmetry is lost. Hence, gravitational radiation can be emitted before percolation occurs. Secondly, the quick distribution of latent heat causes a global reheating. As a consequence, the nucleation rate turns-off after a very short time, and the bubble growth slows down. We have taken these facts into account to calculate the spectrum of GWs in a realistic scenario for deflagrations. 
We have shown that the usual assumptions for the turbulent fluid, namely, the validity of the Kolmogoroff spectrum and the short duration of turbulence in comparison to the Hubble time, apply to the case of deflagration bubbles. We have also derived an analytical approximation for the efficiency factor $\kappa\left(\alpha, v_{w}\right)$ for the deflagration case. In previous works this factor was either calculated numerically or using rough estimations. This analytical approximation makes the treatment of the deflagration case simpler, and will be particularly helpful for including the GW calculation in numerical computations of the phase transition.

We have used the results of Ref. [8] for the GW spectrum from primordial turbulence. There, it is assumed that the frequency $\omega$ of a gravitational wave is determined by the wavenumber of the source mode that generates it, i.e., $\omega=k$, rather than being determined by the characteristic frequency of that mode, $\omega_{L}=2 \pi / \tau_{L}$. As a consequence, the GW spectrum inherits the characteristic wavelength of the source, and the peak is at $\omega_{p} \sim$ $L_{S}^{-1}$. This is correct for a stochastic and statistically homogeneous source of short duration. Recently [9], it was argued that the turbulent source lasts long enough so that it can be treated as stationary. In that case, the resulting GW spectrum would be imprinted with the characteristic frequency of the source, and we would have $\omega_{p} \sim \omega_{S}$. However, the duration of turbulence is on the order of the characteristic turnover time $\tau_{S} \sim \omega_{S}^{-1}$ (since the duration of the stirring source is shorter). Therefore, the characteristic frequency and the duration of the source of GWs are related by $\omega_{S} \tau_{S}=\mathcal{O}(1)$, and the typical frequency of the GWs is given by the typical wave number of the source [27].

The GW spectrum depends on quantities such as $v_{w}, \dot{f}_{b}$, and $d$, which require a numerical computation of the phase transition for a more accurate evaluation. We will address such computation elsewhere [26]. Nevertheless, our estimations show that GWs generated in a strongly first-order electroweak phase transition might be detected by LISA. The parameter values for which this is possible are roughly constrained by $T_{*} \gtrsim 100 \mathrm{GeV}, d \gtrsim 10^{-2} H_{*}^{-1}$, $v_{w} \gtrsim 10^{-2}, \alpha \gtrsim 0.1$. Weakly first-order phase transitions correspond in general to smaller values of $d / H_{*}^{-1}, v_{w}$ and $\alpha$. In that case, the spectrum will have a smaller amplitude and the characteristic frequency will be away from the peak sensitivity of LISA.

\section{Acknowledgments}

I thank T. Kahniashvili for useful discussions. This work was supported in part by Universidad Nacional de Mar del Plata, Argentina, grant EXA 365/07, and by FONCyT grant PICT 33635.

[1] R. Apreda, M. Maggiore, A. Nicolis and A. Riotto, Nucl. Phys. B 631, 342 (2002); A. Nicolis, Class. Quant. Grav. 21, L27 (2004); C. Grojean and G. Servant, Phys. Rev. D 75, 043507 (2007).

[2] M. S. Turner and F. Wilczek, Phys. Rev. Lett. 65, 3080 (1990); A. Kosowsky, M. S. Turner and R. Watkins, Phys. Rev. D 45, 4514 (1992); Phys. Rev. Lett. 69, 2026 (1992); A. Kosowsky and M. S. Turner, Phys. Rev. D 47, 4372 (1993).

[3] S. J. Huber and T. Konstandin, arXiv:0806.1828 [hep-ph].

[4] C. Caprini, R. Durrer and G. Servant, Phys. Rev. D 77, 124015 (2008) arXiv:0711.2593 [astro-ph]]. 
[5] M. Kamionkowski, A. Kosowsky and M. S. Turner, Phys. Rev. D 49, 2837 (1994).

[6] A. Kosowsky, A. Mack and T. Kahniashvili, Phys. Rev. D 66, 024030 (2002).

[7] A. D. Dolgov, D. Grasso and A. Nicolis, Phys. Rev. D 66, 103505 (2002).

[8] C. Caprini and R. Durrer, Phys. Rev. D 74, 063521 (2006).

[9] G. Gogoberidze, T. Kahniashvili and A. Kosowsky, Phys. Rev. D 76, 083002 (2007).

[10] T. Kahniashvili, G. Gogoberidze and B. Ratra, arXiv:0802.3524 [astro-ph].

[11] P. J. Steinhardt, Phys. Rev. D 25, 2074 (1982).

[12] G. D. Moore, JHEP 0003, 006 (2000); P. John and M. G. Schmidt, Nucl. Phys. B 598, 291 (2001) [Erratum-ibid. B 648, 449 (2003)].

[13] B. H. Liu, L. D. McLerran and N. Turok, Phys. Rev. D 46, 2668 (1992); A. E. Nelson, D. B. Kaplan and A. G. Cohen, Nucl. Phys. B 373, 453 (1992).

[14] A. Megevand and A. D. Sanchez, Phys. Rev. D 77, 063519 (2008).

[15] I. Affleck, Phys. Rev. Lett. 46, 388 (1981); A. D. Linde, Nucl. Phys. B 216, 421 (1983) [Erratum-ibid. B 223, 544 (1983)]; Phys. Lett. B 100, 37 (1981).

[16] T. DeGrand and K. Kajantie, Phys. Lett. B 147, 273 (1984); K. Kajantie and H. KurkiSuonio, Phys. Rev. D 34, 1719 (1986); K. Enqvist, J. Ignatius, K. Kajantie and K. Rummukainen, Phys. Rev. D 45, 3415 (1992); P. Y. Huet, K. Kajantie, R. G. Leigh, B. H. Liu and L. D. McLerran, Phys. Rev. D 48, 2477 (1993); A. Megevand, Int. J. Mod. Phys. D 9, $733(2000)$.

[17] J. Ignatius, K. Kajantie, H. Kurki-Suonio and M. Laine, Phys. Rev. D 49, 3854 (1994); M. Gyulassy, K. Kajantie, H. Kurki-Suonio and L. D. McLerran, Nucl. Phys. B 237 (1984) 477; H. Kurki-Suonio, Nucl. Phys. B 255, 231 (1985).

[18] A. F. Heckler, Phys. Rev. D 51, 405 (1995); A. Megevand, Phys. Rev. D 64, 027303 (2001).

[19] A. Megevand, Phys. Lett. B 642, 287 (2006).

[20] A. Megevand, Phys. Rev. D 69, 103521 (2004).

[21] A. Megevand and F. Astorga, Phys. Rev. D 71, 023502 (2005).

[22] L. D. Landau and E. M. Lifshitz, Fluid Mechanics (Pergamon Press, New York, 1989).

[23] M. Maggiore, Phys. Rept. 331, 283 (2000).

[24] C. Caprini and R. Durrer, Phys. Rev. D 65, 023517 (2001) arXiv:astro-ph/0106244.

[25] W.-M. Yao et al., J. Phys. G 33, 1 (2006).

[26] A. Mégevand and A. D. Sánchez (work in progress).

[27] C. Caprini, R. Durrer and R. Sturani, Phys. Rev. D 74, 127501 (2006). 